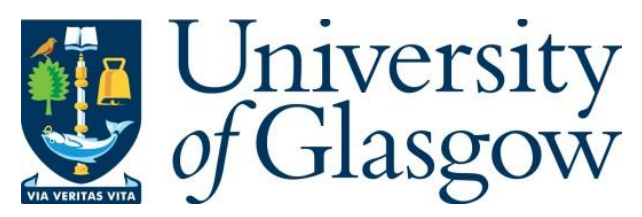

Akhmet'ev, P. M., Candelaresi, S. and Smirnov, A. Y. (2017) Calculations for the practical applications of quadratic helicity in MHD. Physics of Plasmas, 24(10), 102128.

There may be differences between this version and the published version. You are advised to consult the publisher's version if you wish to cite from it.

http://eprints.gla.ac.uk/202963/

Deposited on: 29 January 2020

Enlighten - Research publications by members of the University of Glasgow http://eprints.gla.ac.uk 


\title{
Calculations for the Practical Applications of Quadratic Helicity in MHD
}

\author{
Petr M. Akhmet'ev, ${ }^{1,2}$ Simon Candelaresi, ${ }^{3}$ and Alexandr Yu Smirnov ${ }^{2,4}$ \\ ${ }^{1}$ National Research University Higher School of Economics, Moscow, Russia \\ ${ }^{2}$ IZMIRAN, Troitsk, Moscow region, Russia \\ ${ }^{3}$ Division of Mathematics, University of Dundee, Dundee, DD1 4HN, UK \\ ${ }^{4}$ National University of Science and Technology MISiS, Moscow, 119049, Russia
}

\begin{abstract}
For the quadratic helicity $\chi^{(2)}$ we present a generalization of the Arnol'd inequality which relates the magnetic energy to the quadratic helicity, which poses a lower bound. We then introduce the quadratic helicity density using the classical magnetic helicity density and its derivatives along magnetic field lines. For practical purposes we also compute the flow of the quadratic helicity and show that for an $\alpha^{2}$-dynamo setting it coincides with the flow of the square of the classical helicity. We then show how the quadratic helicity can be extended to obtain an invariant even under compressible deformations. Finally, we conclude with the numerical computation of $\chi^{(2)}$ which show cases the practical usage of this higher order topological invariant.
\end{abstract}

PACS numbers: 51.60.+a, 52.20.-j, 52.25.Xz, 52.30.Cv, 95.30.Qd

\section{INTRODUCTION}

Modern models of non-linear dynamos (e.g. $\left.{ }^{1-11}\right)$, laboratory plasmas (e.g. $\left.{ }^{12,13}\right)$, the solar magnetic field (e.g. ${ }^{14-22}$ ) and other magnetohydrodynamics (MHD) problems (e.g. ${ }^{23-28}$ ) are based on magnetic helicity conservation. Magnetic helicity has a simple geometrical interpretation: by the Arnol'd theorem it measures the pairwise asymptotic linking of magnetic field lines ${ }^{29}$, i.e. it is an invariant of ideal MHD.

Such topological invariants are by their definition conserved under an ideal evolution with vanishing magnetic resistivity where the magnetic field evolves under a Lie-transport (e.g. $\left.{ }^{30,31}\right)$. Approximately ideal conditions exist in various astrophysical settings, like the solar corona where the dynamics of the plasma is dominated by the magnetic pressure with negligible contributions from the hydrostatic pressure gradients.

Additionally, in ideal MHD there exist several topological invariants, which are deduced from the pairwise asymptotic linking numbers of magnetic field lines: the quadratic helicities $^{32}$ and higher momenta of helicity ${ }^{33}$. It has been suggested that such topological invariants give rise to additional constraints on the evolution of a magnetized plasma $\left(\right.$ e.g. $\left.{ }^{34-36}\right)$.

We investigate the properties of these invariants and, in analogy to the magnetic helicity, try to answer the following question: "Is it possible to use the quadratic helicities as non-linear restrictions in MHD problems?". Our results can be used to to further study e.g. the application of higher momenta of helicity in describing nonlinear dynamo saturation, mentioned in ${ }^{37}$. Here we give the definitions and properties of the quadratic helicities and conclude with a practical example for which we compute one of the helicities numerically.

Helicity density is a function on the 4-dimensional space of the ordered pairs of magnetic field lines $\left\{\left(L_{i}, L_{j}\right)\right\}$, which is determined by the Arnol'd asymptotic linking number $h\left(L_{i}, L_{j}\right)^{38}$. The quadratic helicities $\chi^{(2)}, \chi^{[2]}$ are the two (non-central) second momenta of the function $h\left(L_{i}, L_{j}\right)$. The definitions are given in section II.

In this paper we study only the quadratic helicity $\chi^{(2)}$, but in section II, we will refer to $\chi^{[2]}$ and results from ${ }^{32,37,39}$. In section III a generalized Arnol'd inequality for the quadratic helicity is introduced. There we prove that the upper bound of the quadratic helicity is well defined using only the magnetic field $\boldsymbol{B}$. In section IV we propose a formula for $\chi^{(2)}$ using local data (the magnetic helicity density $(\boldsymbol{A}, \boldsymbol{B})$ and its directional derivative along the vector $\boldsymbol{B}$ ). This formula is rather complicated, but its analogy to the formula for magnetic helicity provides an excellent base for applying $\chi^{(2)}$ in practice. Since $\chi^{(2)}$ is not conserved under a general smooth deformation (diffeomorphism) in section $\mathrm{V}$ we give a generalization of $\chi^{(2)}$ that is invariant also under diffeomorphisms with homogeneous density change. For the sake of practical applications, in section VI we present the numerical evaluation of $\chi^{(2)}$ for a linked magnetic field. In section VII we prove that the analog of the helicity flux exists for the quadratic helicity. This result shows that the quadratic helicity can be practically applied.

\section{QUADRATIC MAGNETIC HELICITIES FOR THIN MAGNETIC TUBES}

The quadratic helicities $\chi^{(2)}, \chi^{[2]}$ are defined for a magnetic field $\boldsymbol{B}$ inside a bounded domain $\Omega \subset \mathbb{R}^{3}$, where $\boldsymbol{B}$ is tangent to the boundary of the domain ${ }^{32,37}$. Assume that the magnetic field is represented by a large, but finite number of magnetic tubes $\Omega_{i}$, then the quadratic helicity $\chi^{(2)}$ is well-defined by the formula:

$$
\chi^{(2)}(\boldsymbol{B})=\sum_{i, j, k} \frac{\Phi_{i}^{2} \Phi_{j} \Phi_{k} n\left(L_{j}, L_{i}\right) n\left(L_{i}, L_{k}\right)}{\operatorname{vol}\left(\Omega_{i}\right)},
$$

where $L_{i}, L_{j}, L_{k}$ is a non-ordered collection of central lines of the (thin) magnetic tubes $\Omega_{i}, \Omega_{j}, \Omega_{k}$ with magnetic fluxes $\Phi_{i}, \Phi_{j}, \Phi_{k}$ through their cross-sections. In each collection of 3 tubes one magnetic tube $\Omega_{i}$ is singled out (marked). Two collections of 3 tubes are different even if the tubes coincide, but a different tube is singled out. In equation (1) $n\left(L_{j}, L_{i}\right)$ and $n\left(L_{i}, L_{k}\right)$ are pairwise linking coefficients of central lines of the corresponding magnetic tubes and $\operatorname{vol}\left(\Omega_{i}\right)$ are the volumes filled by the magnetic tubes.

With the same formalism, the quadratic helicity $\chi^{[2]}$ is defined by the following formula where the sum is over non- 
ordered pairs of thin magnetic tubes:

$$
\chi^{[2]}(\boldsymbol{B})=\sum_{i, j} \frac{\Phi_{i}^{2} \Phi_{j}^{2} n^{2}\left(L_{i}, L_{j}\right)}{\operatorname{vol}\left(\Omega_{i}\right) \operatorname{vol}\left(\Omega_{j}\right)} .
$$

We now clarify equation (1) by using the asymptotic ergodic Hopf invariant of magnetic lines (for the definition of the asymptotic Hopf invariant of magnetic lines see e.g. ${ }^{29}$ ). We assume, for simplicity, that the thin magnetic tubes $\Omega_{i}$, $\Omega_{j}, \Omega_{k}$ consist of closed magnetic field lines, each of which is defined as a parallel shift of the central line of the corresponding tube. Assume that the absolute value $|\boldsymbol{B}|$ of the magnetic field is constant along each magnetic line. Then the value of the asymptotic Hopf invariant $h\left(L_{i}, L_{j}\right)$ of a pair of magnetic lines $L_{i} \subset \Omega_{i}$ and $L_{j} \subset \Omega_{j}$ is calculated by the formula:

$$
h\left(L_{i}, L_{j}\right)=\left|\boldsymbol{B}_{i}\right|\left|\boldsymbol{B}_{j}\right| n\left(L_{i}, L_{j}\right)\left|L_{i}\right|^{-1}\left|L_{j}\right|^{-1},
$$

where $\left|L_{i}\right|$ and $\left|L_{j}\right|$ are lengths of the corresponding magnetic lines and we use the relation $\left|\boldsymbol{B}_{i}\right| S_{i}=\Phi_{i}$ for the magnetic flux with the cross sectional area $S_{i}$ of the magnetic tube $\Omega_{i}$ and $\operatorname{vol}\left(\Omega_{i}\right)=\left|L_{i}\right| S_{i}$.

The quadratic helicity $\chi^{(2)}\left(\Omega_{i} ; \Omega_{j} \cup \Omega_{k}\right)$ over the domain $\Omega=\Omega_{i} \cup \Omega_{j} \cup \Omega_{k}$ with a marked magnetic tube $\Omega_{i}$ is calculated as the result of integrating the function $h\left(L_{i}, L_{j}\right) h\left(L_{i}, L_{k}\right)$ over the domain $\Omega$. With the assumption that the function $h\left(L_{i}, L_{j}\right) h\left(L_{i}, L_{k}\right)$ is constant in $\Omega$; as the result of the integration, we get the value:

$$
\begin{aligned}
\chi^{(2)}\left(\Omega_{i} ; \Omega_{j} \cup \Omega_{k}\right)= & \left|\boldsymbol{B}_{i}\right|^{2}\left|\boldsymbol{B}_{j}\right|\left|\boldsymbol{B}_{k}\right| n\left(L_{i}, L_{j}\right) \\
& \times n\left(L_{i}, L_{k}\right)\left|L_{i}\right|^{-2}\left|L_{j}\right|^{-1}\left|L_{k}\right|^{-1} \\
& \times \operatorname{vol}\left(\Omega_{i}\right) \operatorname{vol}\left(\Omega_{j}\right) \operatorname{vol}\left(\Omega_{k}\right) .
\end{aligned}
$$

The remaining two equalities for marked tubes $\Omega_{j}, \Omega_{k}$ are analogous. After taking the sum and a corresponding transformation, equation (4) coincides with the corresponding term in equation (1). Hence, the quadratic helicity $\chi^{(2)}$ (also the quadratic helicity $\chi^{[2]}$ ) is defined analogously to the asymptotic Hopf invariant; the only difference is the following. Instead of a Gaussian linking number, which is a combinatorial invariant of the order 1 (in the sense of V.A. Vassiliev), one uses a combinatorial invariant of the order 2. Definitions and properties of finite-type invariants (Vassiliev invariants) of links can be found in e.g. ${ }^{40}$.

Let us note, that the values of formula (2) are not changed with respect to a subdivision of magnetic tubes into a collection of thinner tubes $\Omega_{i, k}^{\prime}=\gamma_{i, k} \Omega_{i}$ and $\Omega_{j, l}^{\prime}=\gamma_{j, l} \Omega_{j}$, with scaling factors $\gamma_{i, k}$ with $\sum_{k} \gamma_{i, k}=1$. For example, if a magnetic tube $\Omega_{i}$ is divided into $N_{i}$ thinner parallel magnetic tubes $\Omega_{i, k}^{\prime}$ then we get:

$$
\begin{aligned}
\sum_{k} \operatorname{vol}\left(\Omega_{i, k}^{\prime}\right) & =\operatorname{vol}\left(\Omega_{i}\right) \\
\sum_{k} \Phi_{i, k}^{\prime} & =\Phi_{i} \\
n\left(L_{i, k}^{\prime}, L_{j, l}^{\prime}\right) & =n\left(L_{i}, L_{j}\right) .
\end{aligned}
$$

With such a subdivision, equation (2) can be rewritten for an $N_{i}$ in the following way:

$$
\begin{aligned}
\chi^{[2]}(\boldsymbol{B}) & =\sum_{i, j} \sum_{k, l} \frac{\Phi_{i, k}^{\prime 2} \Phi_{j, l}^{\prime 2} n^{2}\left(L_{i, k}^{\prime}, L_{j, l}^{\prime}\right)}{\operatorname{vol}\left(\Omega_{i, k}^{\prime}\right) \operatorname{vol}\left(\Omega_{j, l}^{\prime}\right)} \\
& =\sum_{i, j} n^{2}\left(L_{i}, L_{j}\right) \sum_{k} \frac{\Phi_{i, k}^{\prime 2}}{\operatorname{vol}\left(\Omega_{i, k}^{\prime}\right)} \sum_{l} \frac{\Phi_{j, l}^{\prime 2}}{\operatorname{vol}\left(\Omega_{j, l}^{\prime}\right)} \\
& =\sum_{i, j} n^{2}\left(L_{i}, L_{j}\right) \sum_{k} \frac{\gamma_{i, k}^{2} \Phi_{i}^{2}}{\gamma_{i, k} \operatorname{vol}\left(\Omega_{i}\right)} \sum_{l} \frac{\gamma_{j, l}^{2} \Phi_{j}^{2}}{\gamma_{j, l} \operatorname{vol}\left(\Omega_{j}\right)} \\
& =\sum_{i, j} \frac{\Phi_{i}^{2} \Phi_{j}^{2} n^{2}\left(L_{i}, L_{j}\right)}{\operatorname{vol}\left(\Omega_{i}\right) \operatorname{vol}\left(\Omega_{j}\right)},
\end{aligned}
$$

The right hand side of the equation does not depend on the number of subdivisions $N_{i}$.

\section{THE ARNOL'D INEQUALITY FOR $\chi^{(2)}$ AND ITS GENERALIZATIONS}

The following inequality:

$$
U_{(2)}(\boldsymbol{B}) \geq C|\chi(\boldsymbol{B})|, \quad U_{(2)}(\boldsymbol{B})=\int(\boldsymbol{B}, \boldsymbol{B}) \mathrm{d} \Omega,
$$

where $(.,$.$) denotes the scalar product, \chi$ the magnetic helicity and $C$ a positive constant, is called the Arnol'd inequality ${ }^{29}$. This inequality relates the magnetic energy (on the left hand side) to the magnetic helicity (on the right hand side). The constant $C>0$ does not depend on the magnetic field $\boldsymbol{B}$, but on the geometrical properties of the domain $\Omega$, which is assumed to be a compact domain supporting $\boldsymbol{B}$.

Using the model for $\boldsymbol{B}$ from $^{41}$ we prove the inequality

$$
U_{(k)}(\boldsymbol{B}) \geq C \chi^{[2]}(\boldsymbol{B})
$$

is not valid for $k<1$ with an arbitrary fixed $C>0$, with

$$
U_{(k)}(\boldsymbol{B})=\int|\boldsymbol{B}|^{k} \mathrm{~d} \Omega, \quad k>0 .
$$

Consider a magnetic field $B$ inside a thin closed magnetic tube $\Omega \subset \mathbb{R}^{3}$ without the thinner concentric magnetic tube $\Omega_{\varepsilon}$ of radius $\varepsilon>0$. The magnetic field $\boldsymbol{B}$ is tangent to both boundary components of $\Omega_{\varepsilon}$. The component of $\boldsymbol{B}$ that is parallel to the central line (which is cut-out) is constant (it is equal to 1). The meridional component of $\boldsymbol{B}$, around the central line, is proportional to $r^{\alpha}$, where $r$ is the distance from a point to the central line. The parameter $\alpha<0$ determines the intensity of the meridional component of $\boldsymbol{B}$, but makes no contribution to the integral magnetic flow trough the crosssection of the magnetic tube $\Omega_{\varepsilon}$, which is perpendicular the the central line.

In the limit $\lim _{r \rightarrow \infty}$ of equation (7) with $\alpha \in(0,-\infty)$, the right and left hand side of equation (7) tend to $+\infty$. In the case of $k<1$ there exists a value $\alpha$, for which the right hand side of the inequality is infinite, but the left hand side is finite.

The quadratic helicity is not continuous with respect to $C^{1}$ small deformations of $\boldsymbol{B}$. In a magnetic tube ergodic domains 
of magnetic field lines are not stable with respect to small $C^{1}$-deformations ${ }^{42,43}$; the quadratic helicity is destroyed by such deformations. When ergodic domains of magnetic lines are destroyed inside the common ergodic domain $\Omega^{\prime}$, magnetic helicity remains fixed, the quadratic helicity $\chi^{(2)}(\boldsymbol{B})$ decreases down to its lower bound, which is equal to the square of the magnetic helicity normalized by the volume of the domain: $\frac{\chi^{2}(\boldsymbol{B})}{\operatorname{vol}\left(\Omega^{\prime}\right)}$.

Here we present a generalization of the Arnol'd inequality (6), using the idea from ${ }^{44}$. The new inequality estimates magnetic energies for $k=6$ and $k=\frac{3}{2}$ using the quadratic helicity $\chi^{(2)}$.

Let $\boldsymbol{B}$ be a magnetic field in a bounded domain $\Omega \subset \mathbb{R}^{3}$, which is tangent to the boundary of the domain. The following inequality is then satisfied:

$$
\left(\frac{\pi}{16}\right)^{\frac{2}{3}} U_{(6)}^{\frac{1}{3}}(\boldsymbol{B}) U_{\left(\frac{3}{2}\right)}^{\frac{4}{3}}(\boldsymbol{B}) \geq \sup _{\boldsymbol{B}^{\prime} ; \varepsilon} \chi^{(2)}\left(\boldsymbol{B}^{\prime}\right) \geq \chi^{(2)}(\boldsymbol{B}),(8)
$$

where $\varepsilon>0$ is an arbitrary infinitesimally small positive constant, $\sup _{\boldsymbol{B}^{\prime} ; \varepsilon} \chi^{(2)}\left(\boldsymbol{B}^{\prime}\right)$ is the upper boundary over arbitrary magnetic fields $\boldsymbol{B}^{\prime}$, which are $\varepsilon$-closed in $C^{1}$-topology to the initial magnetic field $\boldsymbol{B}$. The right hand side of the inequality (8) is an invariant under a smooth volume-preserving transformation of the domain $\Omega$.

Proof. Observe that

$$
\chi^{(2)}(\boldsymbol{B}) \leq \int(\boldsymbol{A}, \boldsymbol{B})^{2} \mathrm{~d} \Omega \leq \int \boldsymbol{B}^{2} \rho^{2} \mathrm{~d} \Omega,
$$

where

$$
\|\boldsymbol{A}(\boldsymbol{x})\| \leq \rho(\boldsymbol{x})=\frac{1}{4 \pi} \int_{\Omega} \frac{\|\boldsymbol{B}\|}{\|\boldsymbol{x}-\boldsymbol{y}\|^{2}} \mathrm{~d} \boldsymbol{y} .
$$

Using arguments of ${ }^{29}$ (III, proof of theorem 5.3, the HardyLittlewood inequality), by Hölder's inequality with $p=\frac{1}{3}$ and $q=\frac{2}{3}$ we obtain:

$$
\begin{aligned}
& \int \boldsymbol{B}^{2} \rho^{2} \mathrm{~d} \Omega \leq U_{(6)}^{\frac{1}{3}}(\boldsymbol{B})\left(\int \rho^{3} \mathrm{~d} \Omega\right)^{\frac{2}{3}} \\
& \leq\left(\frac{\pi}{16}\right)^{\frac{2}{3}} U_{(6)}^{\frac{1}{3}}(\boldsymbol{B})\left(U(\boldsymbol{B})_{\left(\frac{3}{2}\right)}\right)^{\frac{4}{3}} \cdot \square
\end{aligned}
$$

\section{QUADRATIC MAGNETIC HELICITY DENSITY}

The magnetic helicity is computed through the magnetic helicity density $(\boldsymbol{A}, \boldsymbol{B})$. The quadratic helicities, however, admit no densities which makes the invariants hard to calculate. In this section we introduce the analogue of magnetic helicity density for the quadratic helicity $\chi^{(2)}$.

Denote the magnetic helicity density by $h=(\boldsymbol{A}, \boldsymbol{B})$ in $\Omega$. A magnetic line $L_{i}$ is equipped with the magnetic flow parameter $\tau$, this parameter determines the magnetic flow in $\Omega$ generated by the vector field $\boldsymbol{B}(\boldsymbol{x})$.
Along each magnetic line $L_{i}$ define the decomposition $(\boldsymbol{A}(\tau), \boldsymbol{B}(\tau))=\bar{h}+\delta f(\tau)$, where $\bar{h}$ is a mean value of $(\boldsymbol{A}(\tau), \boldsymbol{B}(\tau))$ along the line $L_{i}$ (which is well-defined for almost arbitrary magnetic lines $L_{i}$ ) and $\delta f(\tau)$ is a variation with zero mean value. The definition of $\bar{h}$ is clear for closed magnetic lines, for open magnetic lines the Arnol'd approach is based on the Birkhoff theorem.

The quadratic helicity $\chi^{(2)}(\boldsymbol{B})$ is defined in $^{32}$ as the result of the integration of the functions $\bar{h}^{2}$ over the domain $\Omega$, where the function $\bar{h}^{2}(\boldsymbol{x})$ is constant on each magnetic line $L_{i}$. With that definition we can rewrite the expression for $\chi^{(2)}$ using the magnetic vector potential $\boldsymbol{A}$ and avoid computing mutual linking numbers like $n\left(L_{i}, L_{j}\right)$.

We now calculate the square of the mean value of $(\boldsymbol{A}, \boldsymbol{B})$ on a magnetic field line $L_{i}$ starting at point $\boldsymbol{x}$ with limit $T, 0 \leq \tau \leq T$ as $\left(m_{\boldsymbol{x}, T}[(\boldsymbol{A}, \boldsymbol{B})]\right)^{2}$ for sufficiently large $T$. In the limit $T \rightarrow+\infty$ we obtain the mean value. This makes $m_{\boldsymbol{x}, T}[(\boldsymbol{A}, \boldsymbol{B})]$ the mean value of $(\boldsymbol{A}, \boldsymbol{B})$ on $L_{i}$ over the parameter $\tau$. Then we integrate the function $\left(m_{\boldsymbol{x}, T=+\infty}[(\boldsymbol{A}, \boldsymbol{B})]\right)^{2}$ in the domain $\Omega$. As result we obtain $\chi^{(2)}(\boldsymbol{B})$. Obviously, $\left(m_{\boldsymbol{x}, T=+\infty}[(\boldsymbol{A}, \boldsymbol{B})]\right)^{2}$ does not change if we choose a different starting point $\boldsymbol{x} \in L_{i}$, because the mean value over an infinite magnetic line does not depend on the integral over a finite segment. and we can take $\left(m_{\boldsymbol{x}, T=+\infty}[(\boldsymbol{A}, \boldsymbol{B})]\right)^{2}$ in the formula.

This method has a major defect. The integration is in general highly sensitive on the starting point $\boldsymbol{x}$ of the magnetic line. This is especially true for chaotic magnetic field lines. In case we integrate along a random collection of curves, which are uniformly distributed in $\Omega$, the integral coincides not with $\chi^{(2)}(\boldsymbol{B})$, but with $\chi^{2}(\boldsymbol{B}) / \operatorname{vol}(\Omega)$, because the integral tends to its minimal possible value. With this the minimal value of $\chi^{(2)}$ coincides with the lower bound ${ }^{32}$.

Here we present an alternative way to calculate $\chi^{(2)}(\boldsymbol{B})$. In order to simplify the proofs we assume that each magnetic line $L_{i}$ is closed, but the method can be applied to the general case. For simplicity we now omit the indices $i$. Consider the formula for the mean square of the magnetic helicity along a magnetic line starting at position $\boldsymbol{x}$ :

$$
m_{\boldsymbol{x}, T}\left[(\bar{h}(\boldsymbol{x})+\delta f(\boldsymbol{x}))^{2}\right]=\bar{h}^{2}+m_{\boldsymbol{x}, T}\left[\delta f^{2}\right] .
$$

Here, the term $2 m_{\boldsymbol{x}, T}[\bar{h} \delta f]$ is trivial along lines and is omitted, because $\bar{h}$ is a constant and $\delta f(\boldsymbol{x}), \boldsymbol{x} \in L$ is the term with zero mean value. The integral over the domain $\Omega$ of the left hand side of formula (9) is easy to calculate:

$$
\iiint m_{\boldsymbol{x}, T}\left[(\bar{h}+\delta f)^{2}\right] \mathrm{d} \Omega=\iiint(\boldsymbol{A}, \boldsymbol{B})^{2} \mathrm{~d} \Omega,
$$

because $\bar{h}(\boldsymbol{x})+\delta f(\boldsymbol{x})=\left.(\boldsymbol{A}, \boldsymbol{B})\right|_{\boldsymbol{x}}, \boldsymbol{x} \in L$, and

$$
\iiint m_{\boldsymbol{x}, T}\left[(\boldsymbol{A}, \boldsymbol{B})^{2}\right] \mathrm{d} \Omega=\iiint(\boldsymbol{A}, \boldsymbol{B})^{2} \mathrm{~d} \Omega .
$$

Our goal is to calculate the integral

$$
\iiint m_{\boldsymbol{x}, T}\left[\delta f^{2}\right] \mathrm{d} \Omega .
$$


To calculate the term $m_{\boldsymbol{x}, T}\left[(\delta f)^{2}\right]$ we present the double integration over a subdomain in the Cartesian product of the magnetic line starting at $\boldsymbol{x}$. Consider a mean integral over $\tau \in[0, T]:$

$$
\frac{1}{T} \int_{0}^{T}(\bar{h}+\delta f(\tau)) \int_{0}^{\tau} \frac{\mathrm{d}\left(\delta f\left(\tau_{1}\right)\right)}{\mathrm{d} \tau_{1}} \mathrm{~d} \tau_{1} \mathrm{~d} \tau,
$$

where $\tau_{1}$ and $\tau$ are the curve parameters of the magnetic flow with $0 \leq \tau_{1} \leq \tau \leq T$ on $L$ starting at $\boldsymbol{x}$. By taking the average over $\tau$ we assume that $T \rightarrow+\infty$.

Using the equation:

$$
m_{\boldsymbol{x}}\left[\text { p.v. } \int_{-\infty}^{0} \frac{\mathrm{d}\left(\delta f\left(\tau_{1}\right)\right)}{\mathrm{d} \tau_{1}} \mathrm{~d} \tau_{1}\right]=0,
$$

where the integral over $\tau_{1}$ is calculated by its principal value (p.v.), or, as the Cesàro mean. From (12) we then get:

$$
\begin{aligned}
& m_{\boldsymbol{x}, \tau}\left[\delta f^{2}\right]= \\
& m_{\boldsymbol{x}}\left[\frac{1}{T} \int_{0}^{T} \int_{-\infty}^{\tau}(\bar{h}+\delta f(\tau)) \frac{\mathrm{d} \delta f\left(\tau_{1}\right)}{\mathrm{d} \tau_{1}} \mathrm{~d} \tau_{1} \mathrm{~d} \tau\right] .
\end{aligned}
$$

If we put $\tau_{1}=\tau+\tau_{2}$ in the equation we obtain:

$$
\begin{aligned}
& m_{\boldsymbol{x}, \tau}\left[\delta f^{2}\right]= \\
& m_{\boldsymbol{x}}\left[\frac{1}{T} \int_{0}^{T} \int_{-\infty}^{0}(\bar{h}+\delta f(\tau)) \frac{\mathrm{d} \delta f\left(\tau_{2}+\tau\right)}{\mathrm{d} \tau_{2}} \mathrm{~d} \tau_{2} \mathrm{~d} \tau\right]
\end{aligned}
$$

or, if we change the order of integration:

$$
\begin{aligned}
& m_{\boldsymbol{x}, \tau}\left[\delta f^{2}\right]= \\
& m_{\boldsymbol{x}}\left[\text { p.v. } \int_{-\infty}^{0} \frac{1}{T} \int_{0}^{T}(\bar{h}+\delta f(\tau)) \frac{\mathrm{d} \delta f\left(\tau_{2}+\tau\right)}{\mathrm{d} \tau_{2}} \mathrm{~d} \tau \mathrm{d} \tau_{2}\right] .
\end{aligned}
$$

The first term in the integration vanishes, i.e.

$$
\int_{-\infty}^{0} \frac{1}{T} \int_{0}^{T} \bar{h} \frac{\mathrm{d} \delta f\left(\tau_{2}+\tau\right)}{\mathrm{d} \tau_{2}} \mathrm{~d} \tau \mathrm{d} \tau_{2}=0 .
$$

Let us now define the function $\phi$ as

$$
\phi\left(\tau_{2}, \boldsymbol{x}\right)=\frac{1}{T} \int_{0}^{T} \delta f(\tau) \frac{\mathrm{d} \delta f\left(\tau_{2}+\tau\right)}{\mathrm{d} \tau_{2}} \mathrm{~d} \tau .
$$

By the ergodicity we may replace in the integral $-\infty$ by $+\infty$ which results into

$$
\begin{aligned}
m_{\boldsymbol{x}, \tau}\left[\delta f^{2}\right] & =m_{\boldsymbol{x}}\left[\text { p.v. } \int_{-\infty}^{0} \phi\left(\tau_{2}, \boldsymbol{x}\right) \mathrm{d} \tau_{2}\right] \\
& =-m_{\boldsymbol{x}}\left[\text { p.v. } \int_{0}^{+\infty} \phi\left(\tau_{2}, \boldsymbol{x}\right) \mathrm{d} \tau_{2}\right] .
\end{aligned}
$$

Equation (16) is clear: for an arbitrary $\boldsymbol{x} \in L$ define the function $\int_{0}^{\tau} \delta f\left(\tau_{2}\right) \mathrm{d} \tau_{2}=\varphi(\tau, \boldsymbol{x})$ for $\tau \geq 0$ with the boundary condition $\varphi(0, \boldsymbol{x})=0$. This function is a branch of the scalar potential of the vector $\boldsymbol{A}$ along the magnetic line $L$ with starting point $\boldsymbol{x}$. The function $\varphi(\tau, \boldsymbol{x})$ is well-defined by the boundary condition $\varphi(0, \boldsymbol{x})=0$. Take the field $C(\boldsymbol{x})$, such that $m[\varphi(\tau, \boldsymbol{x})+C(\boldsymbol{x})]=0$ on $L$. Denote $\varphi(\tau, \boldsymbol{x})+C(\boldsymbol{x})$ by $\bar{\varphi}(\tau, \boldsymbol{x})$. With that, and because $C(\boldsymbol{x})=-m[\varphi(\tau, \boldsymbol{x})]$, we obtain $C(\boldsymbol{x})=\left.\bar{\varphi}(\tau, \boldsymbol{x})\right|_{\tau=0}$.

The equation (16) can be replaced by:

$$
\begin{aligned}
& m_{\boldsymbol{x}, \tau_{2}}\left[\delta f^{2}\right]=-m_{\boldsymbol{x}, \tau}\left[\frac{1}{T} \int_{0}^{T} \bar{\varphi}(\tau, \boldsymbol{x}) \frac{\mathrm{d} \delta f\left(\tau_{2}\right)}{\mathrm{d} \tau_{2}} \mathrm{~d} \tau\right] \\
& \text { p.v. }[\bar{\varphi}]=0 .
\end{aligned}
$$

Then the formula for the quadratic helicity is presented as:

$$
\chi^{(2)}=\iiint(\boldsymbol{A}, \boldsymbol{B})^{2} \mathrm{~d} \Omega-\iiint m_{\boldsymbol{x}, \tau}\left[\delta f^{2}\right] \mathrm{d} \Omega,
$$

with the second term given by equation (16) or (17).

Finally, let us prove the boundary condition for $\phi\left(\tau_{2}, \boldsymbol{x}\right)$ in (16):

$$
\iiint \phi(0, \boldsymbol{x}) \mathrm{d} \Omega=0
$$

Recall,

$$
\begin{aligned}
& (\boldsymbol{A}(\tau), \boldsymbol{B}(\tau))=\bar{h}+\delta f(\tau), \\
& \frac{\mathrm{d}(\boldsymbol{A}(\tau), \boldsymbol{B}(\tau))}{\mathrm{d} \tau}=\frac{\mathrm{d} \delta f(\tau)}{\mathrm{d} \tau} .
\end{aligned}
$$

We get $\phi(0, \boldsymbol{x})=(\boldsymbol{A}, \boldsymbol{B}) \frac{\mathrm{d}(\boldsymbol{A}, \boldsymbol{B})}{\mathrm{d} \tau}=\frac{1}{2} \frac{\mathrm{d}(\boldsymbol{A}, \boldsymbol{B})^{2}}{\mathrm{~d} \tau}$ and

$$
\iiint \frac{\mathrm{d}(\boldsymbol{A}, \boldsymbol{B})^{2}}{\mathrm{~d} \tau} \mathrm{d} \Omega=0,
$$

because the magnetic flow preserves the integral $\iint(\boldsymbol{A}, \boldsymbol{B})^{2} \mathrm{~d} \Omega$.

Analogously, we get

$$
\begin{gathered}
\iiint \frac{\mathrm{d}^{2} \phi(0, \boldsymbol{x})}{\mathrm{d} \tau^{2}} \mathrm{~d} \Omega=\iiint(\boldsymbol{A}(\tau), \boldsymbol{B}(\tau)) \frac{\mathrm{d}^{3}(\boldsymbol{A}(\tau), \boldsymbol{B}(\tau))}{\mathrm{d} \tau^{3}} \mathrm{~d} \Omega= \\
\iiint 3 \frac{\mathrm{d}^{2}(\boldsymbol{A}(\tau), \boldsymbol{B}(\tau))}{\mathrm{d} \tau^{2}} \frac{\mathrm{d}(\boldsymbol{A}(\tau) \boldsymbol{B}(\tau))}{\mathrm{d} \tau} \mathrm{d} \Omega+ \\
\iiint(\boldsymbol{A}(\tau), \boldsymbol{B}(\tau)) \frac{\mathrm{d}^{3}(\boldsymbol{A}(\tau), \boldsymbol{B}(\tau))}{\mathrm{d} \tau^{3}} \mathrm{~d} \Omega= \\
\iiint \frac{\mathrm{d}^{2}}{\mathrm{~d} \tau^{2}}\left[(\boldsymbol{A}(\tau), \boldsymbol{B}(\tau)) \frac{\mathrm{d}(\boldsymbol{A}(\tau), \boldsymbol{B}(\tau))}{\mathrm{d} \tau}\right] \mathrm{d} \Omega=0 .
\end{gathered}
$$

By analogous arguments, for $k \geq 0$, we get:

$$
\iiint \frac{\mathrm{d}^{2 k} \phi(0, \boldsymbol{x})}{\mathrm{d} \tau^{2 k}} \mathrm{~d} \Omega=0 .
$$

By construction,

$$
\frac{\mathrm{d}(\boldsymbol{A}(\tau), \boldsymbol{B}(\tau))}{\mathrm{d} \tau}=(\operatorname{grad}(\boldsymbol{A}, \boldsymbol{B}), \boldsymbol{B}) .
$$

Denote

$$
\iiint \phi(\tau, \boldsymbol{x}) \mathrm{d} \Omega=\Phi(\tau), \quad \tau \geq 0 .
$$


Let us decompose this function into the Fourier integral, using (19), (21):

$$
\Phi(\tau)=\text { p.v. } \int_{0}^{+\infty} b(y) \sin (y \tau) \mathrm{d} y .
$$

In this formula we collect all possible Fourier coefficients $b(y)$ over the spectrum $y$ with elementary harmonics $\sin (y \tau)$, each elementary harmonic satisfies equation (21) and for $\tau \rightarrow+\infty$ tends to its principal value

$$
\text { p.v. } \lim _{\tau \rightarrow+\infty} \frac{b(y)}{y}[1-\cos (y \tau)]=\frac{b(y)}{y} .
$$

The required second term in (18) is calculated as the principal value of $\Phi(\tau), \tau \rightarrow+\infty$ over $\tau$, or as the Cesàro mean value $\langle\Phi\rangle$ of $\Phi(\tau)$ by the following formula:

$$
\langle\Phi\rangle=\left[\int_{0}^{+\infty} \frac{b(y)}{y} \mathrm{~d} y\right] .
$$

Let us assume that the spectral densities $b(y)$ have a compact support which belongs to the segment $y \in\left[\delta_{0}, \delta_{1}\right]$, $\delta_{0}>0$. This assumption gives simplifications of the problem for $y \rightarrow+\infty$ (very long complicated magnetic lines), and for $y \rightarrow 0+$ (higher harmonics of the magnetic spectrum), in the case of closed magnetic lines with a lower estimation of curvature by a positive constant the condition is satisfied. Let us calculate values, using equation (22),

$$
\frac{\mathrm{d} \Phi(\tau)}{\mathrm{d} \tau}, \quad \frac{\mathrm{d}^{3} \Phi(\tau)}{\mathrm{d} \tau^{3}}, \quad \ldots, \quad \frac{\mathrm{d}^{2 k-1} \Phi(\tau)}{\mathrm{d} \tau^{2 k-1}}, \quad \ldots
$$

for $\tau=0, k \geq 1$ by the formulas:

$$
\begin{aligned}
\left.\frac{\mathrm{d}^{2 k-1} \Phi(\tau)}{\mathrm{d} \tau^{2 k-1}}\right|_{\tau=0} & =\left.\iiint(\boldsymbol{A}, \boldsymbol{B}) \frac{\mathrm{d}^{2 k}(\boldsymbol{A}, \boldsymbol{B})}{\mathrm{d} \tau^{2 k}}\right|_{\tau=0} \mathrm{~d} \Omega \\
& =(-1)^{k} \int_{\delta_{0}}^{\delta_{1}} y^{2 k-1} b(y) \mathrm{d} y .
\end{aligned}
$$

From equation (24) we obtain $\Phi(0)=0$. The left hand sides of the formulas are calculated by data, while the right hand sides are the total collection of momenta of the required function $\frac{b(y)}{y}$ in the integral (23). By this collection the integrals (16), and (23) are calculated as follows.

For the parameters $y \geq \delta_{0}, a>0,\left(a \gg \delta_{0}^{-2}\right)$ we have the limit $\lim _{a \rightarrow+\infty}\left(1-\exp \left(-a y^{2}\right)\right)=1$. But we can also write this expression using its Taylor expansion:

$$
1 \approx 1-\exp \left(-a y^{2}\right)=\sum_{k=1}^{\infty}(-1)^{k+1} \frac{a^{k} y^{2 k}}{k !} .
$$

With that the integral (23) is calculated as following:

$$
\begin{aligned}
\langle\Phi\rangle & =\int_{\delta_{0}}^{\delta_{1}} \frac{b(y)}{y} \mathrm{~d} y \\
& =\sum_{k=1}^{\infty}(-1)^{k+1} \frac{a^{k}}{k !} \int_{\delta_{0}}^{\delta_{1}} y^{2 k-1} b(y) \mathrm{d} y \\
& =-\left.\sum_{k=1}^{\infty} \frac{a^{k}}{k !} \frac{\mathrm{d}^{2 k-1} \Phi(\tau)}{\mathrm{d} \tau^{2 k-1}}\right|_{\tau=0} .
\end{aligned}
$$

This formula (of $(1-\exp (-a))$-type, $a>0$ ) is taken in the case $a$ is sufficiently large.

Let us consider the simplest example. Assume that the magnetic field contains the closed magnetic line $L$. The cyclic covering over this magnetic line is the real line $\tilde{L}=$ $(-\infty,+\infty)$ with the period $2 \pi$. Assume that $(\boldsymbol{A}, \boldsymbol{B})=$ $\bar{h}+\sin (\tau), \tau \in \tilde{L}$. Then the first term of the integral (18) is $\bar{h}^{2}+\frac{1}{2}$, the second term is $\frac{1}{2}$ and the quadratic helicity over $L$ equals to $\bar{h}^{2}$.

Let us calculate the second term in this formula using the function (22). We get:

$$
\begin{gathered}
-\langle\Phi\rangle=m_{x, \tau}\left[\delta f^{2}\right]=-m_{\tau}\left[\int_{0}^{+\infty} \Phi\left(\tau_{2}\right) \mathrm{d} \tau_{2}\right]= \\
- \text { p.v. } \int_{0}^{+\infty} m_{\tau}\left[\int_{0}^{+\infty} \sin (\tau) \cos \left(\tau+\tau_{2}\right) \mathrm{d} \tau\right] \mathrm{d} \tau_{2}= \\
-\frac{1}{2} \text { p.v. } \int_{0}^{+\infty} \sin \left(\tau_{2}\right) \mathrm{d} \tau_{2}=-\frac{1}{2} \text { p.v. } \lim _{\tau_{2} \rightarrow+\infty}\left[1-\cos \left(\tau_{2}\right)\right]=-\frac{1}{2} .
\end{gathered}
$$

Obviously, using the equation (25), because

$$
\begin{gathered}
\left.\frac{\mathrm{d}^{2 k} \Phi}{\mathrm{d} \tau^{2 k}}\right|_{\tau=0}=\frac{(-1)^{k}}{2 \pi} \int_{0}^{2 \pi} \sin ^{2}\left(\tau_{2}\right) \mathrm{d} \tau_{2}, \\
\frac{1}{2 \pi} \int_{0}^{2 \pi} \sin ^{2}\left(\tau_{2}\right) \mathrm{d} \tau_{2}=\frac{1}{2}
\end{gathered}
$$

we get:

$$
\langle\Phi\rangle=\lim _{a \rightarrow+\infty}-\frac{1}{2} \sum_{k=1}^{\infty} \frac{(-1)^{k} a^{k}}{k !}=\frac{1}{2} .
$$

\section{COMPRESSIBILITY EFFECTS ON THE QUADRATIC HELICITY $\chi^{(2)}$}

For many physical applications the underlying fluid or gas is described as being compressible, rather than incompressible. Such systems include those in plasma physics and MHD with applications in astrophysics and fusion science. While in their current definitions $\chi^{(2)}$ and $\chi^{[2]}$ are invariant under incompressible fluid deformations, they need to be modified to assure their invariance under a general diffeomorphism that includes compression. We show that by simply including the fluid density we obtain quantities that are invariant under a diffeomorphisms that change the density uniformly in space.

$\operatorname{In}^{32}$ (equation (2)) the authors use the square of the average magnetic helicity along a magnetic line $\Lambda^{(2)}(T ; \boldsymbol{x})$. It is defined as

$$
\Lambda^{(2)}(T ; \boldsymbol{x})=\frac{1}{T^{2}}\left(\int_{0}^{T}(\dot{\boldsymbol{x}}(\tau), \boldsymbol{A}(\boldsymbol{x}(\tau))) \mathrm{d} \tau\right)^{2},
$$


with the magnetic vector potential $\boldsymbol{A}$ of the field and the velocity vector $\dot{\boldsymbol{x}}(\tau)=\boldsymbol{B}(\boldsymbol{x}(\tau))$. The integral is taken along a magnetic field line starting at position $\boldsymbol{x}=\boldsymbol{x}(\tau=0)$. With that ${ }^{32}$ wrote the quadratic helicity $\chi^{(2)}$ as

$$
\chi^{(2)}=\limsup _{T \rightarrow \infty} \int \Lambda^{(2)}(T ; \boldsymbol{x}) \mathrm{d} D,
$$

where $D \in \mathbb{R}^{3}$ is a ball of radius $r$. By Birkhoff Theorem the limit in (28) exists for almost arbitrary $\boldsymbol{x}$, see ${ }^{32}$, section 5 . The velocity $\dot{\boldsymbol{x}}(\tau)$ along the magnetic field line is equivalent to $\boldsymbol{B}$ at position $\boldsymbol{x}(\tau)$.

Assume that magnetic lines are closed, this gives a simplification of the proof. With that we can identify equation (28) as the squared of the average magnetic helicity density along a magnetic flux line of integration length $T$. For the sake of a compact notation we will write the integrand of equation (28) as the scalar product of the magnetic vector potential and the magnetic field, i.e. $(\boldsymbol{A}, \boldsymbol{B}) \Leftrightarrow(\dot{\boldsymbol{x}}(\tau), \boldsymbol{A}(\boldsymbol{x}(\tau)))$.

We now investigate the cases of diffeomorphisms stretching the coordinate system along and across the magnetic field lines assuming a fluid density of $\rho_{0}=1$ before applying the mapping. For a stretching along the magnetic field lines by a factor of $\lambda$, the density changes to $\rho=\lambda^{-1} . \quad B$ is invariant under such a transformation because the integral magnetic flow is invariant and the cross-section is fixed. The total length of the field line at parameter $\tau=T$ changes, but the mean of the magnetic helicity density does not, hence $\overline{(\boldsymbol{A}, \boldsymbol{B})} \mapsto \overline{(\boldsymbol{A}, \boldsymbol{B}) \rho^{-1}}$. So, the function $\overline{(\boldsymbol{A}, \boldsymbol{B}) \rho^{-1}}$ is frozen in. We now substitute $(\boldsymbol{A}, \boldsymbol{B})$ by $(\boldsymbol{A}, \boldsymbol{B}) \rho^{-1}$ in equation (28) which adds a factor of $\rho^{-1}$ in equation (29):

$$
\Lambda_{\rho}^{(2)}(T ; \boldsymbol{x})=\frac{1}{T^{2}}\left(\int_{0}^{T} \frac{(\dot{\boldsymbol{x}}(\tau), \boldsymbol{A}(\boldsymbol{x}(\tau)))}{\rho(\boldsymbol{x}(\tau)))} \mathrm{d} \tau\right)^{2} .
$$

However, with $\rho$ also the measure $\mathrm{d} D$ changes to $\rho \mathrm{d} D$. We get the following formula for $\chi^{(2)}$ in the compressed fluid:

$$
\chi_{\rho}^{(2)}=\iint \Lambda_{\rho}^{2} \rho \mathrm{d} D
$$

For a stretching across the magnetic field lines by a factor of $\lambda$ along both directions the density changes as $\rho=\lambda^{-2}$. In order to conserve magnetic flux across comoving surfaces the magnetic field changes according to $\lambda^{-2} \boldsymbol{B}=\rho \boldsymbol{B}$. Again, $\overline{(\boldsymbol{A}, \boldsymbol{B}) \rho^{-1}}$ is invariant. The integral measure changes according to $\lambda^{-2}$.

In both cases the additional factor of $\rho$ in the two integrands cancel if $\rho$ does not depend on space. In that case we obtain a quadratic magnetic helicity that is invariant under (homogeneously) density changing diffeomorphisms.

From the changes of $\chi^{(2)}$ for general transformations, we can conclude that $\chi^{(2)}$ is not a function of the magnetic helicity $h$ and does form part of invariants suggested by ${ }^{43}$. If it was true, then for two fields with the same helicity, the quadratic helicity would be the same. Here we construct a simple counter example. Take a field $\boldsymbol{B}_{1}$ with helicity $h_{1}$. Construct a second field $\boldsymbol{B}_{2}$ via a topology conserving transformation of $\boldsymbol{B}_{1}$, then $h_{1}=h_{2}$. If this transformation is not volume conserving, then, in general, the quadratic helicities are different, i.e. $h_{1} \neq h_{2}$.

\section{NUMERICAL CALCULATIONS OF $\chi_{\rho}^{(2)}$}

For practical applications, like MHD simulations, equations (30) and (31) can be computed numerically. In order to evaluate the integral in equation (30) we need to trace magnetic field lines (streamlines), while for the integral in equation (31) we integrate in space.

\section{A. Hopf Link}

As simple test case we apply our calculations on the Hopf link which consists of two magnetic flux tubes with a finite width which are interlinked. In our construction they both have the radius 1 and a tube diameter (FWHM) of 0.28284 . Their centers are located at $(0,0,0)$ and $(1,0,0)$ with surface normals of $(0,0,1)$ and $(0,1,0)$. The magnetic vector potential for the first ring is given as

$$
\begin{aligned}
\zeta^{1} & =e^{\left(-\left(x^{2}+y^{2}+z^{2}-2 \sqrt{x^{2}+y^{2}}+1\right) \eta\right)} / \eta \\
A_{x}^{1} & =\frac{\sqrt{\pi} \sqrt{\eta} x \zeta^{1} \operatorname{erf}(\sqrt{\eta} z) e^{\left(\eta z^{2}\right)}}{2 \sqrt{x^{2}+y^{2}}} \\
A_{y}^{1} & =\frac{\sqrt{\pi} \sqrt{\eta} y \zeta^{1} \operatorname{erf}(\sqrt{\eta} z) e^{\left(\eta z^{2}\right)}}{2 \sqrt{x^{2}+y^{2}}} \\
A_{z}^{1} & =\frac{1}{2} \zeta^{1},
\end{aligned}
$$

while for the second it is given as

$$
\begin{aligned}
\zeta^{2} & =e^{\left(-\left((x-1)^{2}+y^{2}+z^{2}-2 \sqrt{(x-1)^{2}+z^{2}}+1\right) \eta\right)} / \eta \\
A_{x}^{2} & =\frac{\sqrt{\pi} \sqrt{\eta}(x-1) \zeta^{2} \operatorname{erf}(\sqrt{\eta} y) e^{\left(\eta y^{2}\right)}}{2 \sqrt{(x-1)^{2}+z^{2}}} \\
A_{y}^{2} & =\frac{1}{2} \zeta^{2} \\
A_{z}^{2} & =\frac{\sqrt{\pi} \sqrt{\eta} z \zeta^{2} \operatorname{erf}(\sqrt{\eta} y) e^{\left(\eta y^{2}\right)}}{2 \sqrt{(x-1)^{2}+z^{2}}},
\end{aligned}
$$

with the total vector potential

$$
\boldsymbol{A}_{\mathrm{Hopf}}=\boldsymbol{A}^{1}+\boldsymbol{A}^{2} .
$$

From this we can write the magnetic field for the two flux rings as

$$
\begin{aligned}
& B_{x}^{1}=-\frac{\sqrt{\pi} \eta^{\frac{3}{2}} y z \zeta^{1} \operatorname{erf}(\sqrt{\eta} z) e^{\left(\eta z^{2}\right)}+\eta y \zeta^{1}}{\sqrt{x^{2}+y^{2}}} \\
& B_{y}^{1}=\frac{\sqrt{\pi} \eta^{\frac{3}{2}} x z \zeta^{1} \operatorname{erf}(\sqrt{\eta} z) e^{\left(\eta z^{2}\right)}+\eta x \zeta^{1}}{\sqrt{x^{2}+y^{2}}} \\
& B_{z}^{1}=0
\end{aligned}
$$




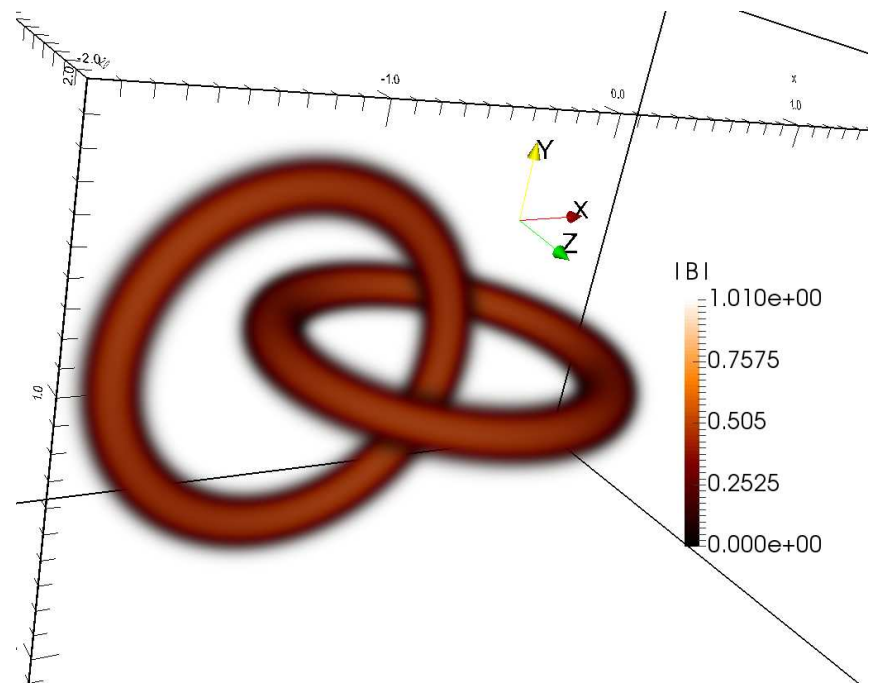

FIG. 1. Volume rendering of the magnetic field strength for the Hopf link used in our calculations.

and

$$
\begin{aligned}
& B_{x}^{2}=\frac{\sqrt{\pi} \eta^{\frac{3}{2}} y z \zeta^{2} \operatorname{erf}(\sqrt{\eta} y) e^{\left(\eta y^{2}\right)}+\eta z \zeta^{2}}{\sqrt{x^{2}+z^{2}-2 x+1}} \\
& B_{y}^{2}=0 \\
& B_{z}^{2}=-\frac{\left(\sqrt{\pi} \sqrt{\eta} y \operatorname{erf}(\sqrt{\eta} y) e^{\left(\eta y^{2}\right)}+1\right) \eta(x-1) \zeta^{2}}{\sqrt{x^{2}+z^{2}-2 x+1}}
\end{aligned}
$$

with the total magnetic field as their sum

$$
\boldsymbol{B}_{\mathrm{Hopf}}=\boldsymbol{B}^{1}+\boldsymbol{B}^{2} .
$$

A representation of this Hopf link is shown in Fig. 1.

\section{B. Change of coordinates}

We apply five different simple homeomorphisms which change the grid density uniformly and compare them to the initial grid. By changing the grid we also need to transform the magnetic field $\boldsymbol{B}$ and the magnetic vector potential $\boldsymbol{A}$. We do this by applying the pull-back on the corresponding differential forms:

$$
\begin{aligned}
\alpha_{0} & =A_{x} \mathrm{~d} x+A_{y} \mathrm{~d} y+A_{z} \mathrm{~d} z \\
\beta_{0} & =B_{x} \mathrm{~d} y \wedge \mathrm{d} z+B_{y} \mathrm{~d} z \wedge \mathrm{d} x+B_{z} \mathrm{~d} x \wedge \mathrm{d} y \\
\alpha & =F^{*}\left(\alpha_{0}\right) \\
\beta & =F^{*}\left(\beta_{0}\right),
\end{aligned}
$$

with the mapping $F$.

We stretch and compress the grid in various directions and perform the integral (30) and (31) on the deformed field. For simplicity we choose factors of 2 for the deformations. These deformations we the abbreviate using the short notation e.g. uvw $=(2,0.5,1)$, which implies a transformation of $(u, v, w)=(2 x, 0.5 y, 1 z)$, with the new coordinates

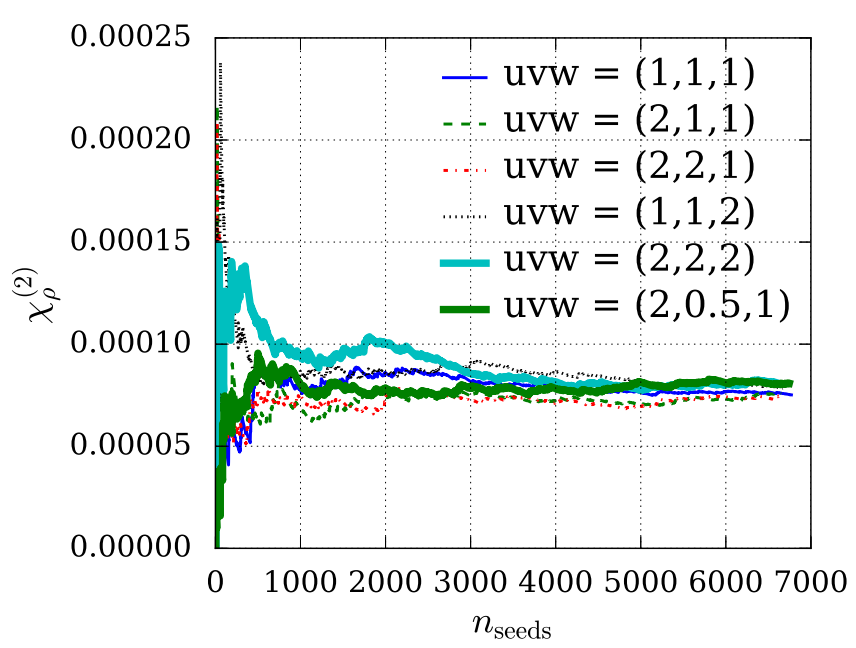

FIG. 2. The modified quadratic helicity $\chi_{\rho}^{(2)}$ in dependence of the number of seed points for the integration of equation (31) for different homeomorphisms. We clearly see the convergence to the same value of $\chi_{\rho}^{(2)}$. Here we denote the mapping $F$ by the new coordinates $u, v$ and $w$, where e.g. $\mathrm{uvw}=(2,0.5,1)$ corresponds to $(u, v, w)=(2 x, 0.5 y, 1 z)$.

$(u, v, w)$. In order to minimize selection bias we choose as starting points for our integration a set of ca. 7000 homogeneously distributed points within a sphere of radius 2 centered at the origin. Those points are then transformed according to the homeomorphism. A larger number of such points will provide us with a more accurate value for $\chi_{\rho}^{(2)}$. In Fig. 2 we plot the value for $\chi_{\rho}^{(2)}$ for different grid deformations and number of seed points for the integration. We clearly see convergence to the same value which confirms our result that the modified $\chi_{\rho}^{(2)}$ is invariant under a homeomorphism which homogeneously changes the grid density.

\section{QUADRATIC MAGNETIC HELICITY FLOW}

$\mathrm{By}^{32}$, Theorem 2, the quadratic magnetic helicity $\chi^{(2)}$ admits a continuous variation in the case of $C^{2}$-small flows (vector of flows $\frac{\partial B}{\partial t}$ in the domain $\Omega$ with its first and second partial derivatives small). $\mathrm{In}^{43}$ is claimed, that KAM-theory proves (for generic functionals) the analogous statement for $C^{k}$-small flows, $k>3$.

Let us calculate, a contribution of the advection of magnetic lines to the main term of the variation of $\chi^{(2)}$. Let us consider $C^{2}$-small perturbations $\delta \boldsymbol{B}$ and $\delta \boldsymbol{A}$ :

$$
\boldsymbol{B} \mapsto \boldsymbol{B}+\delta \boldsymbol{B}, \quad \boldsymbol{A} \mapsto \boldsymbol{A}+\delta \boldsymbol{A} ; \quad \boldsymbol{\nabla} \times \delta \boldsymbol{A}=\delta \boldsymbol{B}
$$

Using Corollary 1 from $^{32}$, the first-order gauge transformation 
of the equation (28) is represented by:

$$
\begin{aligned}
\Lambda^{(2)}(T ; \boldsymbol{x}) \mapsto & \Lambda^{(2)}(T ; \boldsymbol{x})+ \\
& \frac{2}{T^{2}} \int_{0}^{T}(\dot{\boldsymbol{x}}(\tau), \delta \boldsymbol{A}(\boldsymbol{x}(\tau))) \mathrm{d} \tau \\
& \times \int_{0}^{T}(\dot{\boldsymbol{x}}(\tau), \boldsymbol{A}(\boldsymbol{x}(\tau)) \mathrm{d} \tau+ \\
& \frac{2}{T}\left(\left.m_{\tau}[\delta \boldsymbol{B}(\boldsymbol{x}(\tau))]\right|_{\tau=T}, \boldsymbol{A}(\boldsymbol{x}(T))\right) \\
& \times \int_{0}^{T}(\dot{\boldsymbol{x}}(\tau), \boldsymbol{A}(\boldsymbol{x}(\tau)) \mathrm{d} \tau .
\end{aligned}
$$

The first extra term corresponds to the gauge term $\delta \boldsymbol{A}$. The second extra term is given by the scalar product of the average $m_{\tau}[\delta \boldsymbol{B}]$ of the gauge vector $\delta \boldsymbol{B}$ over the magnetic line $\boldsymbol{x}(\tau)$ at the point $\boldsymbol{x}(T)$. (This term is given by the second term from the equation in Corollary 1 from $^{32}$.) As the result, in the limit $\tau \rightarrow+\infty$ we get the following expression of the timederivative of the quadratic helicity:

$$
\begin{aligned}
& \frac{\mathrm{d} \chi^{(2)}}{\mathrm{d} t}= \\
& \left.2 \iiint m_{\tau}\left[\left(\frac{\partial \boldsymbol{A}(\boldsymbol{x}(\tau))}{\partial t}, \boldsymbol{B}(\boldsymbol{x}(\tau))\right)\right]\right|_{\tau=\tau_{0}}+ \\
& \left(\left.m_{\tau}\left[\frac{\partial \boldsymbol{B}(\boldsymbol{x}(\tau))}{\partial t}\right]\right|_{\tau=\tau_{0}}, \boldsymbol{A}\left(\boldsymbol{x}\left(\tau_{0}\right)\right)\right) \\
& \times m_{\tau}\left[\left.(\boldsymbol{B}(\boldsymbol{x}(\tau), \boldsymbol{A}(\tau))]\right|_{\tau=\tau_{0}} \mathrm{~d} \Omega .\right.
\end{aligned}
$$

By this property we get that the limit over the parameter $T \rightarrow+\infty$ of formula (28) is uniform with respect to $t-$ variations of the magnetic field $\boldsymbol{B}$. To calculate $\frac{\mathrm{d} \chi^{(2)}}{\mathrm{d} t}$, for $t=t_{0}$, it is sufficient to calculate the first-order derivative in equation (28) over $t$ for a prescribed $T$. In particular, for $\alpha^{2}$ dynamos we get: $\frac{\partial \boldsymbol{A}}{\partial t}=\alpha \boldsymbol{B}$; and, assuming $\boldsymbol{\nabla} \times \boldsymbol{B}=\boldsymbol{k} \times \boldsymbol{B}$, we have: $\frac{\mathrm{d} \chi^{(2)}}{\mathrm{d} t}=4 \alpha k \chi^{(2)}$. This means that the flow of quadratic magnetic helicity $\chi^{(2)}$ for the magnetic field with the $k$-vector coincides with the flow of the square of the helicity $\chi^{2}$.

\section{CONCLUSIONS}

Both, the magnetic helicity and the quadratic helicities, can be calculated from experimental data and be used in various
MHD problems. For that, the main tool and example is the Arnol'd inequality (6), which relates the geometry of magnetic field lines (Gauss linking numbers or asymptotic Hopf invariants) with the magnetic energy. Analogous inequalities relate higher magnetic energies with higher momenta of magnetic helicity (see the formula (8)). This inequality shows that the upper bound of the quadratic helicity for $C^{0}$-closed magnetic fields is limited.

For many problems in MHD one uses not only the total magnetic helicity, but its density. The density of magnetic helicity is defined as $(\boldsymbol{A}, \boldsymbol{B})$. This value is not invariant with respect to transformations of the domain with the magnetic field. To calculate the quadratic helicity $\chi^{(2)}$ equation (18) is proposed. The first term in the formula is easy to calculate. To calculate the second term one needs to know the structure of the ergodic magnetic subdomains in $\Omega$, where each subdomain contains a dense collection of magnetic field lines. An ergodic subdomain can be shrunk to a surface, to a line, or even, to a critical point of $\boldsymbol{B}$. Therefore, quadratic magnetic helicities are much harder to use in applications then the magnetic helicity and equation (29) might be preferred.

We then showed that the quadratic helicity $\chi^{(2)}$ can be extended to be invariant under non-volume preserving diffeomorphisms, as long as they change the density homogeneously. We calculated that quantity numerically for the Hopf link and showed that it is indeed invariant under such diffeomorphisms. This has important applications in various fields of MHD.

\section{ACKNOWLEDGEMENTS}

Petr Akhmet'ev and Alexandr Smirnov are supported in part by the Russian Foundation for Basic Research (grant No. 15-02-01407). Simon Candelaresi acknowledges financial support from the UK's STFC (grant number ST/K000993). We thank the anonymous referee for the improvement suggestions.
${ }^{1}$ U. Frisch, A. Pouquet, J. Léorat, and A. Mazure, J. Fluid Mech. 68, 769 (1975).

${ }^{2}$ H. Ji, Phys. Rev. Lett. 83, 3198 (1999).

3 E. T. Vishniac and J. Cho, Astrophys. J. 550, 752 (2001).

4 A. Brandenburg, W. Dobler, and K. Subramanian, Astron. Nachr. 323, 99 (2002).

5 A. Brandenburg, S. Candelaresi, and P. Chatterjee, Mon. Not. R. Astron. Soc. 398, 1414 (2009).
${ }^{6}$ J. Pietarila Graham, E. G. Blackman, P. D. Mininni, and A. Pouquet, Phys. Rev. E 85, 066406 (2012).

7 F. Del Sordo, G. Guerrero, and A. Brandenburg, Mon. Not. R. Astron. Soc. 429, 1686 (2013).

8 D. Telloni, S. Perri, R. Bruno, V. Carbone, and R. D. Amicis, Astrophys. J. 776, 3 (2013).

9 V. V. Pipin, D. D. Sokoloff, H. Zhang, and K. M. Kuzanyan, Astrophys. J. 768, 46 (2013). 
10 Tziotziou, K., Moraitis, K., Georgoulis, M. K., and Archontis, V., Astron. Astrophys. 570, L1 (2014).

11 A. Brandenburg, G. J. D. Petrie, and N. K. Singh, Astrophys. J. 836, 21 (2017)

12 J. B. Taylor, Phys. Rev. Lett. 33, 1139 (1974).

13 O. Motojima, K. Ida, K. Watanabe, Y. Nagayama, A. Komori, T. Morisaki, B. Peterson, Y. Takeiri, K. Ohkubo, K. Tanaka, and et al., Nucl. Fusion 45, S255 (2005).

${ }^{14}$ M. A. Berger, Geophys. Astro. Fluid 30, 79 (1984).

15 M. A. Berger and A. Ruzmaikin, J. Geophys. Res. 105, 10481 (2000).

${ }^{16}$ N. Kleeorin, D. Moss, I. Rogachevskii, and D. Sokoloff, Astron. Astrophys. 361, L5 (2000).

17 P. Démoulin and M. Berger, Sol. Phys. 215, 203 (2003).

18 J. Chae, Adv. Space Res. 39, 1700 (2007).

19 Y. Guo, M. D. Ding, X. Cheng, J. Zhao, and E. Pariat, Astrophys. J. 779, 157 (2013).

${ }^{20}$ Y. Liu, J. T. Hoeksema, M. Bobra, K. Hayashi, P. W. Schuck, and X. Sun, Astrophys. J. 785, 13 (2014).

${ }^{21}$ P. Romano, F. P. Zuccarello, S. L. Guglielmino, and F. Zuccarello, Astrophys. J. 794, 118 (2014).

${ }^{22}$ Pariat, E., Valori, G., Dmoulin, P., and Dalmasse, K., Astron. Astrophys. 580, A128 (2015).

23 A. Kumar and D. M. Rust, J. Geophys. Res.-Space 101, 15667 (1996).

24 A. Shukurov, D. Sokoloff, K. Subramanian, and A. Brandenburg, Astron. Astrophys. 448, L33 (2006).

25 G. Guerrero, P. Chatterjee, and A. Brandenburg, Mon. Not. R. Astron. Soc. 409, 1619 (2010).

26 A. Pevtsov, M. Berger, A. Nindos, A. Norton, and L. van DrielGesztelyi, Space Sci. Rev. 186, 285 (2014).
27 A. J. B. Russell, A. R. Yeates, G. Hornig, and A. L. WilmotSmith, Phys. Plasmas 22, 032106 (2015).

${ }_{28}$ M. Linkmann and V. Dallas, Phys. Rev. E 94, 053209 (2016).

29 V. Arnol'd and B. Khesin, Applied Mathematical Sciences, 125 (2013).

${ }^{30}$ I. J. D. Craig and A. D. Sneyd, Astrophys. J. 311, 451 (1986).

31 S. Candelaresi, D. Pontin, and G. Hornig, SIAM J. Sci. Comput. 36, B952 (2014).

${ }^{32}$ P. M. Akhmet'ev, Proceedings of the Steklov Institute of Mathematics 278, 10 (2012).

33 R. Komendarczyk, Commun. Math. Phys. 292, 431 (2009).

${ }^{34}$ F. Del Sordo, S. Candelaresi, and A. Brandenburg, Phys. Rev. E 81, 036401 (2010).

35 A. R. Yeates, G. Hornig, and A. L. Wilmot-Smith, Phys. Rev. Lett. 105, 085002 (2010).

36 S. Candelaresi and A. Brandenburg, Phys. Rev. E 84, 016406 (2011).

37 D. Sokoloff, E. Illarionov, and P. Akhmet'ev, Geomagnetism and Aeronomy 57, 123 (2017).

38 V. I. Arnold, Sel. Math. Sov. 5 (1974).

39 D. Sokoloff, P. Akhmet'ev, and E. Illarionov, "Magnetic helicity and higher helicity invariants as constraints for dynamo action," (2017).

40 V. Prasolov and A. Sossinsky, Trans. Math. Monogr. 154 (1997).

${ }^{41}$ P. M. Akhmet'ev, E. A. Kudryavtseva, and A. Y. Smirnov, Magnetohydrodynamics 52, 5 (2016).

42 E. A. Kudryavtseva, Math. Notes 95, 877 (2014).

43 A. Enciso, D. Peralta-Salas, and F. T. de Lizaur, P. Natl. Acad. Sci. USA 113, 2035 (2016).

${ }^{44}$ R. Komendarczyk and I. Volić, Ergodic Theory and Dynamical Systems 36, 832 (2016). 